\title{
Sparking Limits, Cavity Loading, and Bcam Breakup Instability Associated with High-Current rf Linacs
}

\author{
R. J. Faehl \\ Don S. Lemons \\ Lester E. Thode
}

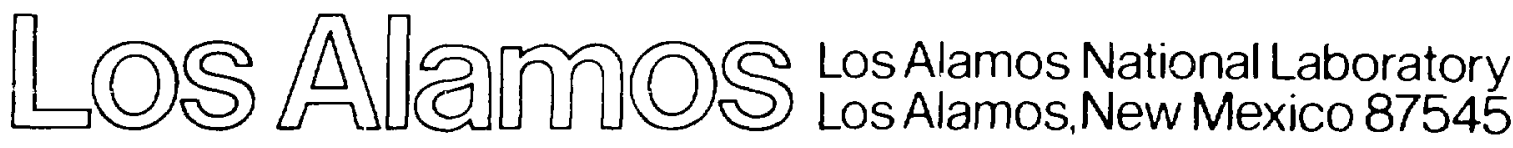


SPARKING LIMITS, CAVITY LOADING, AND BEAM BREAKUP INSTABILITY ASSOCIATED WITH HIGH-CURRENT $\mathrm{r} f$ LINACS

by

R. J. Faehl, Don S. Lemons, and Lester E. Thode

\section{ABSTRACT}

The limitations on high-current $r f$ linacs due to gap sparking, cavity loading, and the beam breakup instability are studied. It appears possible to achieve cavity accelerating gradients as high as $35 \mathrm{MV} / \mathrm{m}$ without sparking. Furthermore, a linear analysis, as well as self-consistent particle simulations of a multipulsed $10 \mathrm{kA}$ beam, indicates that only a negligible small fraction of energy is radiated into nonfundamental cavity modes. Finally, the beam breakup instability is analyzed and found to be able to magnify initial radial perturbations by a factor of no more than about 20 during the beam transit time through a $1 \mathrm{GeV}$ accelerator.

\section{INTRODUCTION}

When a standing-wave radio-frequency ( $r f$ ) linac was first suggested as an alternative to an induction linac for generating a high-current, relativistic electron beam, a number of objections were raised. First, the high accelerating gradients necessary were not possibie because of sparking limitations. Second, beam driven cavity radiation would clearly be catastrophic because the energy source for such radiation is scaled like the square of the charge in a micropulse. Finally, because of the high cavity $Q$, the nonaxisymmetric modes would rapidly deflect the beam to the drift tube wall. We have considered all of these issues in the present report, and we find that none of these objections are valid. Taking into accuunt our previous calculations and particle-in-cell simulations of injection into the first cavity ${ }^{1}$ coupled with the present analysis, a high-current standing-wave $r f$ linac appears possible. 
In Sec. II several criteria on maximum accelerating gradients and associated sparking limits are discussed. In the 50- to 200-MHz range, accelerating gradients of 21 to $35 \mathrm{MV} / \mathrm{m}$ appear possible for well-prepared systems.

The question of axisymmetric beam loading is addressed in Sec. III, both analytically and through particle-in-cell plasma simulation techniques. For multipulse trains, beam driven radiation into unwanted modes is found to be small, provided the cavity dimensions and micropulse width are chosen to avoid higher order modes with frequencies that are exact odd integer multiples of the fundamental. Radiation into transverse electric (TE) modes is also small.

Finally, the question of beam breakup instability is addressed in Sec. IV. Because of the high accelerating gradicnts, the beam becomes stiff quickly and beam deflections are found to be quite small. We do not mean to imply that this type of instability can be ignored, but it does appear to be controllable and is certainly not catastrophic.

\section{ACCELERATING GRADIENTS AND SPARKING LIMITS}

One of the advantages of standing-wave $r f$ over induction linacs is their potential for high accelerating gradients. Gradients in an rf linac are limited only by sparking effects and cavity $Q$ considerations.

Sparking can be caused by charge particle multipazting whenever

$$
T \geq 1 / 2 f
$$

where $T$ is the particle transit time and $f$ is the frequency of the fundamental accelerating frequency. In terms of the peak electric field $E_{z}$, this limit is

$$
E_{z}=\operatorname{md}(2 \pi f)^{2} / 2 e
$$

where $d$ is the cavity length and $m$ and $e$ are respectively the electron mass and charge.

Electron multipacting can be overcome relatively easily by rapidly increasing the field over the limit of Eq. (2). For a PHERMEX ${ }^{2}$ cavity $(\mathrm{d}=2.6 \mathrm{~m}$, $f=50 \mathrm{MHz}$ ), this limit is only $0.728 \mathrm{MV} / \mathrm{m}$.

The upper limit for ion multipacting is, on the other hand, larger by a factor of the ion over electron mass ratio. ${ }^{3}$ The lower limit at which ion type 
sparking actually begins to occur is complicated by cavity surface effects and residual gas pressure. An empirically motivated expression for the lower threshold for jon sparking is given by ${ }^{*}$

$$
f(M H z)=1.643 E_{K}^{2}(M V / m) \exp \left[-8.5 / E_{K}(M V / m)\right]
$$

and

$$
E_{z} \cong K E_{K} / G \quad
$$

where $G$ is a geometrical enhancement factor and $K$ is a factor that depends upon surface conditions and residual gas pressure. For a PHERMEX cavity $G=1.26$, and for a well-prepared system $K \cong 2$ to 3 . Equation (3) is similar to a result obtained by Kilpatrick. 4

An $r f$ cavity should, therefore, operate with an accelerating gradient between the ion sparking limit of Eq. (4) and the electron multipacting limit of Eq. (2). The former is plotted as a function of fundamental frequency $f$ in Fig. 1 .

The energy stored in a cavity is

$$
\mathrm{U}=\frac{1}{2} \varepsilon_{0} \mathrm{E}_{\mathrm{z}}^{2} \pi \mathrm{R}^{2} \mathrm{~d} J_{1}^{2}\left(\chi_{01}\right)
$$

where $K$ is the cavity radius and $J_{0}\left(\chi_{01}\right)=0$. For each micropulse passing through the cavity, the cavity energy is reduced by

$$
\delta \mathrm{U} \cong 0.707 \rho \mathrm{dE}_{\mathrm{z}}
$$

where $\rho$ is the total charge in a single micropulse. Finally, the approximate accelerator length per $\mathrm{GeV}$ is

$$
L(m) \cong 1.414 \times 10^{3} / E_{z}(M V / m)
$$

This length does not include the injector and $\xi o c_{1} ; 4$, , e..ements.

*. J Boyd, private communication to L. E. Thode, June 1981. 
In Tables I through III a summary of the field strength implications are given for $50 \mathrm{MHz}, 100 \mathrm{MHz}$, and $200 \mathrm{MHz}$, respectively. In obtaining these results, we have assumed a one-half wavelength cavity with each micropulse having a peak current of $10 \mathrm{kA}$. The square current pulse width is $\mathrm{T}=0.25 \mathrm{~d} / \mathrm{c}$. Looking at the number of pulses, [U/OU], it is clear that a significant amount

TABLE I

SUMMARY OF FIELD STRENGTH IMPLICATIONS AT $50 \mathrm{mHz}$ WITH $\rho=2.5 \times 10^{-5}$ COULOMB/MICROPULSE

\begin{tabular}{|c|c|c|c|c|c|}
\hline Criteria & $\mathrm{E}_{\mathrm{z}}(\mathrm{MV} / \mathrm{m})$ & $\mathrm{U}(\mathrm{kJ})$ & $\delta \mathrm{U}(\mathrm{kJ})$ & {$[\mathrm{U} / \delta \mathrm{U}]$} & L. (m) \\
\hline $\begin{array}{c}\text { Equation (4) } \\
K=2\end{array}$ & 14 & 11.7 & 0.74 & 15 & 101.0 \\
\hline $\begin{array}{c}\text { Equation } \\
K=3\end{array}$ & 21 & 26.2 & 1.11 & 23 & 67.3 \\
\hline
\end{tabular}

TABLE I I

SUMMARY OF FIELD STRENGTH IMPLICATIONS OF $100 \mathrm{MHz}$ WITH $\rho=1.25 \times 10^{-5}$ COULOMB/MICROPULSE

\begin{tabular}{|c|c|c|c|c|c|}
\hline Criteria & $E_{z}(M V / m)$ & $\mathrm{U}(\mathrm{kJ})$ & $\delta U(k J)$ & {$[\mathrm{U} / \delta \mathrm{U}]$} & $\mathrm{L}(\mathrm{m})$ \\
\hline $\begin{array}{c}\text { Equation } \\
\mathrm{K}=2\end{array}$ & 18 & 2.41 & 0.24 & 10 & 78.6 \\
\hline $\begin{array}{c}\text { Equation } \\
K=3\end{array}$ & 27 & 5.46 & 0.36 & 15 & 52.4 \\
\hline
\end{tabular}

TABLE III

SUMMARY OF FIELD STRE TOTH IMPLICATIONS AT $200 \mathrm{MHz}$ WITH $\rho=6.25 \times 10^{-6}$ COULOMB/MICROPULSE

\begin{tabular}{|c|c|c|c|c|c|}
\hline Criteria & $E_{z}(M V / m)$ & $U(k J)$ & $\delta U(\mathbf{k J})$ & [U/DU] & $L(m)$ \\
\hline $\begin{array}{c}\text { Equation (4) } \\
K=2\end{array}$ & 23 & 0.491 & 0.076 & 6 & 61.5 \\
\hline $\begin{array}{c}\text { Equation (4) } \\
K=3\end{array}$ & 35 & 1.137 & 0.116 & 9 & 40.4 \\
\hline
\end{tabular}


of rf energy must be supplied to each cavity if long pulse trains are desirable. This implies that the cavity $Q$ must be relatively low, i.e., $Q \cong 50$ to 200 . This would have the added advantage of more control on the pulse-to-pulse energy variation and would also provide better energy utilization. Most importantly, the accelerator lengths are far shorter than even the most optimistic induction linac concepts.

\section{AXISYMMETRIC BEAM LOADING}

\section{A. Background}

Cavity loading due to beam driven radiation is a well-known problem in rf linear accelerators. In fact, one of the major criticisms of the highcurrent $\mathrm{rf}$ linac conceft was that beam driven radiation into unwanted modes would be catastrophic because the radiation energy source scales as $\vec{E} \cdot \vec{J}$, or as the charge of a micropulse squared. In particular, enhanced spectral content in unwanted modes not only serves as a sink for beam energy but it can seriously magnify transverse beam emittance. Even worse, the coupling to the nonaxisymmetric $\ell=1$ mode would deflect the beam into the drift tube wall. This last issue of the beam breakup mode ${ }^{5}$ is addressed in Sec. IV. In the present section we have undertaken a quantitative study of high-current driven cavity radiation to indicate both the magnitude and scaling of the axisymmetric beam loading.

A simple right ciicular, cylindrical cavity is employed to represent the essential physics, if not the details of more complicated, realistic cavity shapes. A major advantage of this is that the eigenmodes for such a system are readily calculable. Even though the ideal structure is perturbed by drift tube apertures of radius $r_{d}$, this is a relatively small effect so long as the cavity radius $R$ satisfies $R \gg r_{d}$. As a further simplification, we will consider only single cavities, driven at the fundamental TM frequency. Although coupling of cavities is often done to enhance synchronism, we feel that this more complex configuration can best be illuminated initially by treating the single cavity loading exhaustively.

The study is limited to intense electron beams, which are at least modestly relativistic upon injection into the cavity. In fact, when treating the beam dynamics with simulation, the electrons are constrained to be neither relativistic nor to follow one-dimensional trajectories. Transport of multikiloampere 
beams into $\mathrm{rf}$ cavities, however, does require that they be relativistic enough to avoid space-charge limitations. For 10-kA micropulses this corresponds to about $3 \mathrm{MV} .^{1}$ In the simulations, the transport itself was aided by assumption of a straight solenoidal magnetic guide field. We can include more realistic focusing $\tilde{i}$ ields, but there is no reason for doing so at this juncture.

\section{B. Analytic Model}

The model we use to calculate the response of the cavity modes to the beam is well known. 6 It is not a self-consistent calculation in that the modification of the beam distribution by the cavity fields is not taken into account. However, we find the results to be in good agreement with self-consistent electromagnetic, relativistic, two-dimensional, particle-in-cell simulation results.

In terms of the vector potential $\vec{A}$ and the scalar potential $\phi$, Ampere's law becomes

$$
\nabla^{2} \vec{A}-\frac{1}{c^{2}} \frac{\partial^{2} \vec{A}}{\partial t^{2}}=-\mu_{0} \vec{J}-\frac{1}{c^{2}} \frac{2}{\partial t} \nabla \phi
$$

We have chosen the Coulomb gauge: $\nabla \cdot \vec{A}=0$. The vector potential can be expressed in terms of the cavity eigenmodes $\vec{a}_{\lambda}$,

$$
\vec{A}=\sum_{\lambda} q_{\lambda} \vec{a}_{\lambda}
$$

which satisfy $\nabla^{2} \vec{a}_{\lambda}+\left(w_{\lambda}^{2} / c^{2}\right) \vec{a}_{\lambda}=0$. Substituting Eq. (9) into Eq. (8), multiplying by $\vec{a}_{\lambda}{ }^{\prime}$, and integrating over the cavity volume $\Omega$ yields

$$
\ddot{q}_{\lambda}+w_{\lambda}^{2} q_{\lambda}=\frac{1}{\varepsilon_{0}} \int_{\Omega} \vec{a}_{\lambda} \cdot\left(\vec{J}+\varepsilon_{0} \frac{\partial}{\partial t} \nabla \phi\right) d^{3} x
$$

The 1ast term in Eq. (10) can be rewritten as 


$$
\frac{\partial}{\partial t} \int_{\Omega} \vec{a}_{\lambda} \cdot \nabla \phi d^{3} x=\frac{\partial}{\partial t} \int_{S} \phi \vec{a} \cdot d \vec{s}-\frac{\partial}{d t} \int_{\Omega} \phi \nabla \cdot \vec{a}_{\lambda} d^{3} x .
$$

Because $\phi=0$ on the cavity surface and $\nabla \cdot \vec{a}_{\lambda}=0$ in the cavity volume, this term vanishes, and the time evolution $o:$ a cavity mole is given by

$$
\ddot{q}_{\lambda}+w_{\lambda}^{2} q_{\lambda}=\frac{j_{\lambda}}{\varepsilon_{0}},
$$

where

$$
\mathrm{j}_{\lambda}=\int_{\Omega} \overrightarrow{\mathrm{a}}_{\Omega} \cdot \overrightarrow{\mathrm{J}} \mathrm{d}^{3} \mathrm{x}
$$

In obtaining Eq. (12) we used the property that

$$
\int_{\Omega} \vec{a}_{\lambda} \cdot \vec{a}_{\lambda} \cdot d^{3} x=\delta_{\lambda \lambda}
$$

This normalization yields

$$
\begin{aligned}
a_{z \ell n p}(\theta, r, z) & =\left(\frac{c x_{\ell n}}{R w_{\ell n p}}\right)\left[\frac{1}{R J_{\ell+1}\left(x_{\ell n}\right)}\right]\left[\frac{4}{\pi d\left(1+\delta_{p 0}\right)\left(1+\delta_{\ell 0}\right)}\right]^{1 / 2} \\
& \times \cos (\ell \theta) J_{\ell}\left(x_{\ell n} r / R\right) \cos (p \pi z / d),
\end{aligned}
$$

where

$$
w_{\ell n p}=c\left[\left(\frac{x_{\ell n}}{R}\right)^{2}+\left(\frac{p \pi}{d}\right)^{2}\right]^{1 / 2} .
$$

All other components can be calculated in terms of $a_{z}$. Again, we have defined $R$ as the cavity radius, $d$ as the cavity gap, and $c$ as the speed of light in vacuum. 
Also, $J_{\ell}\left(x_{\ell n}\right)=0$, where $\ell$ is the azimuthal mode number, $n$ is the radial mode number, and $p$ is the axial mode number.

$$
\begin{aligned}
& \text { Defining } \xi_{\lambda}=\dot{q}_{\lambda}+i w_{\lambda} q_{\lambda} \text {, the solution to Eq. (12) is } \\
& \xi_{\lambda}=v_{\lambda} \exp \left[i\left(\phi_{\lambda}+w_{\lambda} t\right)\right]+D_{\lambda} \exp \left(i w_{\lambda} t\right)
\end{aligned}
$$

where ${ }{ }_{\lambda}$ and $\phi_{\lambda}$ are the magnitude and phase of the homogeneous contribution and

$$
D_{\lambda}=\frac{1}{\varepsilon_{0}} \int_{0}^{t} d t^{\prime} j_{\lambda}\left(t^{\prime}\right) \exp \left(-i \omega_{\lambda} t^{\prime}\right)
$$

is from the inhomogeneous contribution. Initially, $v_{\lambda}=0$ except for the fundamental mode. Having the solution for $\xi_{\lambda}$, the mode energy is

$$
\mathrm{U}_{\lambda}=\frac{1}{2} \varepsilon_{0} \xi_{\lambda} \xi_{\lambda}^{\stackrel{*}{*}}
$$

Given a current density distribution $\vec{J}, V_{\lambda}$, and $\phi_{\lambda}$, Eqs. (17) through (19) describe the cavity loading.

As a starting point, we consider the cavity radiation driven by a point charge that enters the cavity at $t=0$ and moves rigidly along the $z$-axis at velocity c. For such a charge the current density is

$$
J_{z}(z, t)=\rho c \delta(z-c t) \delta(r) / 2 \pi r
$$

From Eq. (13) we obtain

$$
\begin{aligned}
j_{\lambda}(t) & =\rho c\left(\frac{c x_{0 n}}{R w_{0 n p}}\right)\left[\frac{1}{R J_{1}\left(x_{0 n}\right)}\right]\left[\frac{2}{\pi d\left(1+\delta_{0 p}\right)}\right]^{1 / 2} \cos (p \pi c t / d) \\
& =\rho c a_{0 \operatorname{mp}}(0,0,0) \cos (p \pi c t / d),
\end{aligned}
$$


where we have dropped the subscript " $z$ " from a. It follows that

$$
\begin{aligned}
D_{\lambda}^{(1)} & =\frac{\rho c}{s_{0}} a_{0 n p}(0,0,0) \int_{0}^{d / c} d t^{\prime} \cos \left(p \pi c t^{\prime} / d\right) \exp \left(-i w t^{\prime}\right) \\
& =\frac{-i \rho}{\varepsilon_{0} x_{0 n_{1}^{j}}\left(x_{0 n}\right)}\left[\frac{2}{\pi d\left(1+\delta_{0 p}\right)}\right]^{1 / 2}\left[1-(-1)^{p} \exp \left(-i w_{\lambda} d / c\right)\right] .
\end{aligned}
$$

Suppose we have a train of point charges separated by a time $2 \pi / w_{x}$, the current density is then

$$
J_{z}(z, t)=\rho c \sum_{n=1}^{N} \delta\left[z-c t+2 \pi(n-1) c / w_{x}\right] \delta(r) / 2 \pi r
$$

It follows that

$$
\begin{aligned}
& D_{\lambda}^{(N)}=\frac{\rho c}{\varepsilon_{0}} a_{0 n p}(0,0,0) \sum_{n=1}^{N} \int_{2 \pi(n-1) / w_{x}}^{d / c+2 \pi(n-1) w_{x}} d t^{\prime} \cos \left\{\frac{p \pi c}{d}\left[t^{\prime}-2 \pi(n-1) / w_{x}\right]\right\} \exp \left(-i w_{\lambda} t^{\prime}\right) \\
& =\frac{\rho c}{\varepsilon_{0}} a_{0 n p}(0,0,0)\left\{\sum_{n=1}^{N} \exp \left[-i 2 \pi(n-1) w_{\lambda} / w_{x}\right]\right) \int_{0}^{d / c} d t^{\prime} \cos (p \pi c t / d) \exp \left(-i w_{\lambda} t^{\prime}\right) \\
& =N \exp \left[-i \pi(N-1) w_{\lambda} / w_{x}\right]\left[\frac{\sin \left(N \pi w_{\lambda} / w_{x}\right)}{N \sin \left(\pi w_{\lambda} / w_{x}\right)}\right] D_{\lambda}^{(1)} \text {. }
\end{aligned}
$$

If we let $w_{x} \rightarrow \infty$ and $N \rightarrow \infty$ while keeping $T=N \cdot 2 \pi / w_{x}$ constant, we get the result for a constant current pulse of width $T$. Thus, a train of micropulses of finite pulse width yields

$$
D_{\lambda}=\exp \left(-i w_{\lambda} T / 2\right)\left[\frac{\sin \left(w_{\lambda} T / 2\right)}{\left(w_{\lambda} T / 2\right)}\right]_{\lambda}(N)
$$


The energy in an initially undriven mode after $\mathrm{N}$ pulses is then

$$
\begin{aligned}
U_{\lambda}= & \frac{2 N^{2} \rho^{2}}{\varepsilon_{0} \pi d\left(1+\delta_{0 p}\right)} \frac{1}{x_{0 n}^{2} J_{1}^{2}\left(x_{0 n}\right)}\left[\frac{\sin \left(N \pi w_{\lambda} / w_{010}\right)}{N \sin \left(\pi w_{\lambda} / w_{010}\right)}\right]^{2}\left[\frac{\sin \left(w_{\lambda} T / 2\right)}{\left(w_{\lambda} T / 2\right)}\right]^{2} \\
& \times\left\{1-(-1)^{p} \cos \left(w_{\lambda} d / c\right)\right]
\end{aligned}
$$

Finally, the energy radiated into the fundamental mode is determined from

$$
\begin{aligned}
D_{010}^{(N)}= & \exp \left[-i w_{010} d / 2 c-i w_{010} T / 2\right] \\
& \times \frac{2 N \rho}{\varepsilon_{0} x_{01} J_{1}\left(x_{01}\right)}\left(\frac{1}{\pi d}\right)^{1 / 2} \sin \left(w_{010} d / 2 c\right)\left[\frac{\sin \left(w_{010} T / 2\right)}{\left(w_{010} T / 2\right)}\right]
\end{aligned}
$$

and is

$$
\begin{aligned}
& \mathrm{U}_{010}=\frac{1}{2} \varepsilon_{0} \mathrm{E}_{z 010}^{2} \pi \mathrm{R}^{2} \mathrm{~d} \mathrm{~J}_{1}^{2}\left(\mathrm{x}_{01}\right)-\frac{2|\rho| \mathrm{NRE} \mathrm{z}_{210}}{\mathrm{x}_{01}} \sin \left(\omega_{010} \mathrm{~d} / 2 \mathrm{c}\right)\left[\frac{\sin \left(\omega_{010} \mathrm{~T} / 2\right)}{\left(w_{010} \mathrm{~T} / 2\right)}\right] \\
& +\frac{2 N^{2} \rho^{2}}{\pi \varepsilon_{0} d} \frac{1}{x_{01}^{2} J_{1}^{2}\left(x_{01}\right)} \cdot\left[\frac{\sin \left(\omega_{010^{T / 2}}\right)}{\left(\omega_{010} T / 2\right)}\right]^{2} \sin ^{2}\left(\omega_{010} d / 2 c\right)
\end{aligned}
$$

where we have chosen the phase $\phi_{010}$ for maximum acceleration and $v_{010}=$ $E_{z 010}(\pi d)^{\frac{2}{2}} R J_{1}\left(x_{01}\right)$.

\section{Analyrical Results}

Our first concern was to check the analytical results against particle-incell simulations. In Fig. 2 the amount of energy radiated into an initially empt; cavity is plotted as a function of the number of micropulses passing through the cavity. The parameters are $\rho=1.74 \times 10^{-5}$ Coulomb/micropulse, $R=2.3 \mathrm{~m}$, and $\mathrm{d}=2.5625 \mathrm{~m}$. The comparison between the analytic result and the "slug" beam is very good. Where a fully self-consistent beam is injected into the cavity, a combination of space-charge and induced fields reflects the beam 
TABLE IV

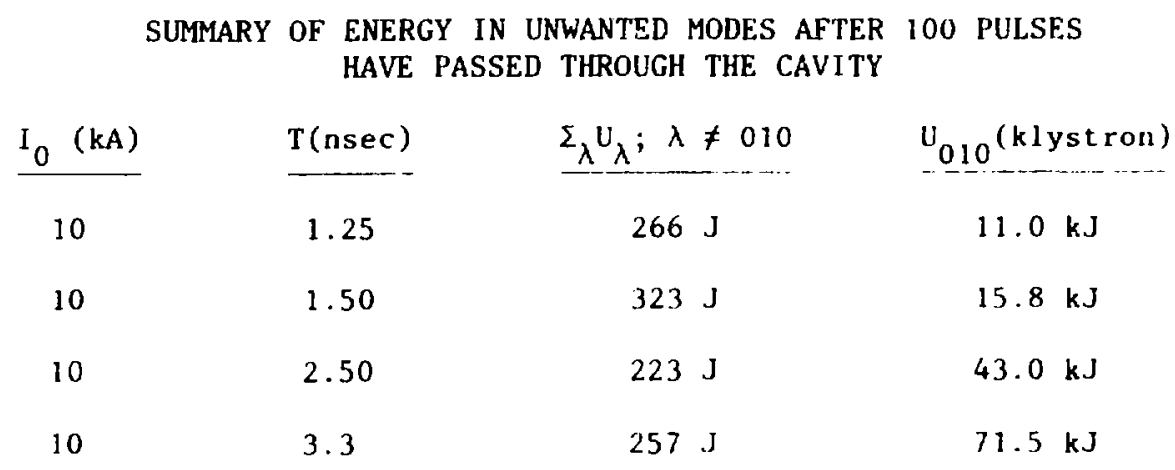

after about seven pulses, that is, the energy in the cavity modes becomes about equal to the kinetic energy of the micropulse. The details of this simulation will be discussed in more detail later, but we feel that the analytic results will yield essentially the correct beam loading for an actual beam.

With some confidence in our result, the energy in unwanted modes was calculated for a $10-\mathrm{kA}$ beam for various micropulse widths after 100 pulses. Again, the cavity dimensions are $R=2.3 \mathrm{~m}$ and $d=2.5625 \mathrm{~m}$. Basically, we find that the energy going into unwonted modes is negligible compared to the energy going into the fundamental. This will be true even for $\ell \neq 0$ modes as long as the cavity is "detuned," that is, $w_{\lambda} / w_{010} \neq$ odd integer. A summary is given in Table IV.

\section{Numesical Simulations}

The simple model derived in the previous section is very useful for calculating the beam loading on the cavity. However, space charge, finite transverse dimensions of the beam, and beam distortion by the cavity fields were neglected in the model. In short, it did not attempt to evaluate the effect of cavity fields on the beam. To study the self-consistent dynamics, we have employed the two-dimensional particle-in-cell code CCUBE. This fully electromagnetic, relativistic simulation code has been used previously in a wide variety of intense non-neutral beam and accelerator studies. The present calculations were performed in cylindrical $(r, z)$ coordinates, with azimuthal symmetry (that is, $\ell=0$ ).

A pill-box, right-circular cavity was used for these simulations. The cavity, when driven, was operated on the ${ }^{T M} 010$ mode. In fact, this cavity 
fundameacal was the dominant mode excited in undriven cavities when a series of beam pulses were injected. The cavity length d was taken to be slightly larger than its radius $R$ with $d / R$ ranging from 1.07 to 1.16. As the calculat ant incie scaled to the TM0 ${ }_{0}$ mode, no absolute dimensions attached are to them. In fact, we are quite interested in PHFRlAX, which operates at 50-Mbz, or similaz highcurrent linacs. For a PHERMFX-l ake cavity, $K=2.3 \mathrm{~m}$ and $\mathrm{d}=2.6 \mathrm{~m}$.

It seems appropriate at this pont to describe the normalizations in the

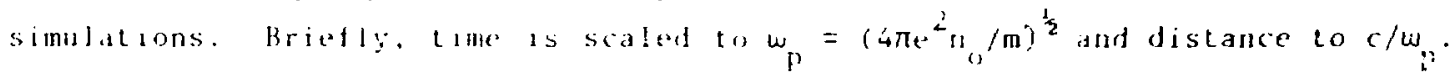
Although the trequency and limens lons ate thus arbitrary, depending on a scaling density no, the current, which is propertiond to fnotr)rdr, is indspendent of no ant thus is an absolute quantity an physucal units. Similiarly potential and energy are scaled to mo ${ }^{2}$ in tht caliulations and are invariant. When fields are quoted, though, the reader should note that they have units of an energy gradient. In general they scall, whth ${ }_{0}^{\frac{1}{*}}$, and a reference tensity has been employed te give the quantities mure practical significance. This deñity was arbitrarily taken to be such that $c / w_{p}=0.05 \mathrm{~m}$.

Because of the complicated dynamics of the full self-consistent loading, a series of cdlculations were performed to explicitly isolate various aspects of the problem. In the first, a sequence of fixed current profiles was propagated through the single cavity. These current "slugs" radiated electromagnetic fields into the cavity but were not in turn acted upcn by the fields. These simulations were closest to the assumptions of the loading model derived in Sec. IIIB. The cavities were not driven so there was no confusion between the radiated field distributions and a preloaded field. The second type of simulation also contained no cavity pre-excitation, but simulation macro-particles were used to construct the injected pulses. Because these pulses were free to respond to the self-excited cavity, space-charge effects and kinetic energy depletion due to $\vec{J} \cdot \vec{E}$ were self-consistently calculated. To facilitate pulse propagation across the cavity, a uniform solenoidal field $B_{z}$, such that $\Omega_{0}=w_{p}$, $\Omega_{0}=|e| B_{z} / m c$, was included. Although imposition of a nonfringing field of this magnitude around a 2.3-m-radius cavity is possible, it is admittedly not practical. For these calculations, it was unnecessary to complicate the beam dynamics with focusing effects, however. Finally, the realistic accelerator problem was treated. We limited the studies to early cavity loading, because cavity loading is most severe before the beam has become too "stiff" $(\gamma \gg 1)$. 
The cavity was driven in the $\mathrm{TM}_{010}$ mode to between $8.5 \mathrm{MV} / \mathrm{m}$ and $15 \mathrm{MV} / \mathrm{m}$ at $50 \mathrm{MHz}$. Maximum peak currents injected were $18.5 \mathrm{kA}$ and minimum, $0.6 \mathrm{kA}$. The former corresponded to an average current of almost $1.4 \mathrm{kA}$.

\section{E. Simulation Results and Discussion}

The base line calculations were slug simulations that can most easily be compared with the analytic model. Several salient features of the model were amenable to simple tests. The model, for inslance, predicted that the total energy radiated into the cavity should vary as the square of the total charge per pulse. This was repeatedly ve;ified for pulses of various radius and longitudinal extent. The spectral distribution of cavity energy, moreover, was identical for pulses of the same physical shape but different density. For pulses injected at the same frequency as the cavity fundamental, the model, moreover, predicted that the fraction of the total cavity energy outside the fundamental would decrease as the number of pulses increased. For a 9.2-kA peak current injection, Fig. 3 shows the fraction of the energy not in $\mathrm{TM}_{010}$ as a function of pulse number in the simulation compared with the theory. As a complement to this, Fig. 2 shows the total radiated energy for this case as a function of pulse number. From these, it is evicient that the pulses are very effectively driving the cavity $\mathrm{TM}_{010}$ mode.

The magnitude of this field is increasing linearly with the number of pulses, so that after 10 pulses, the peak $\mathrm{TM}_{010}$ field has attained a magnitude of $2.8 \mathrm{MV} / \mathrm{m}$. Although this field is in the decelerating phase, it is a significant fraction of the accelerating gradient. This suggests the fascinating prospect of building an rf autoaccelerator. Unlike Friedman's autoaccelerator, in which a long low-voltage beam is used to energize an induction cell which subsequently accelerates a much shorter beam to much higher voltage, the fundamental time scale $\tau=2 \pi / \omega_{010}$ is fixed by the cavity geometry here. The purpose of such a device is not higher voltage, because this is readily attainable in $\mathrm{rf}$ linacs by stacking many cavities in series. Instead, the purpose is to overcome power limitations of existing rf sources. Creating an initial beam by pulse power certainly has limits, but these appear to be in the range of 10s of terawatts as opposed to $10 \mathrm{~s}$ of gigawatts for conventional power supplies. Thus, if a multiterawatt electron beam can be induced to radiate $i$ ts energy into a given cavity mode, it seems quite feasible to operate $r$ linacs in as high a gradient as the cavities can withstand (either Kilpatrick or field emission). 
As mentioned, we have verified that the total energy in the cavity increases as the square of the number of pulses but that the relative fraction outside the fundamental decreases. The excitation of higher order modes, therefore, becomes less important as the number of pulses increases. Evell if the magnitude of fields in these unwanted modes were to remain high, we are confident that loading of the cavities with a frequency dependent absorber could reduce the levels to acceptable values. A more fundamental limitation is depletion of the pulse energy after the gradient reaches sufficient magnitude.

If the kinetic mean energy of an injected pulse is $(\gamma-1) \mathrm{mc}^{2}$, this pulse will lose all its energy once the loaded field has reached a magnitude $E_{z}$, such that

$$
\int_{0}^{d} E_{z} \cos w t d z \geqq(\gamma-1) m c^{2} .
$$

This places an upper bound on $E_{z} ; E_{z}=w(\gamma-1) \mathrm{mc}^{2} /(\mathrm{ec} \sin w d / 2 c)$. Once this gradient is attained, we find that a moving virtual cathode forms on the pulse. By this we mean that a fraction of the beam is reflected, while the rest propagates tilrough the cavity. In a low-current beam for which $\Delta y / \gamma \ll 1$, it is plausible to treat single particle trajectories in which all particles are either transmitted or reflected. For these high-current puises, however, the collective behavior of a virtual cathode is observed whenever the pulse kinetic energy drops below a finite, nonzero value. The reflected portion of the pulse is moving in the opposite direction and so is in an acceleration phase. Because it extracts energy from the cavity, the net energy radiated is reduced from the nonreflecting case. Fig. 4 shows growth of rms $E_{z}$ field at the cavity midpoint for a slug simulation compared with a fully interacting particle one. The field has reached pulse reflecting levels by pulse number seven. Interestingly, even after partial pulse reflection, the $\mathrm{TM}_{010}$ field coitinues to grow. The net efficiency of field generation is clearly reduced, but as Fig. 5 shows, the relative magnitude of the TM 010 mode icreases vis-à-vis any of the nonfundamental and presumably deleterious modes. Actual operation in this fashion may prove undesirable because of breakdown problems associated with residual charge left in the cavity, but it is not ruled out on the basis of the spatial field distribution. 
The spectral distribution of radiated energy is of considerable interest. Unfortunately, exact details of the spectra depend sensitively on details of the cavity structure. As an example, a series of calculations were performed in which the pulse structure and current were identical, with only the width of the cavity varied. Figure 6 shows the $\mathrm{E}_{z}$ power spectrum on axis of three of these calculations. Altho:ıgh the ratio of width to radius of the cavity d/R varied froin only 1.105 to 1.159 , there is a noticeably different spectral structure away from the fundamental $w_{010}=0.052 w_{p}$. In all cases, the $T_{010}$ spectral component after 10 pulses was overwhelmingly dominant and within graphical accuracy at the same level. For a simple pill-box cavity, the next eigenfrequency, which is an integral multiple of the fundamental, is the fifth harmonic. Both analytic and numerical results indicate that this should be the next strongest mode. There is in fact a near coincidence of the $\mathrm{TM}_{033}$ and $\mathrm{TM}_{014}$ modes here. Integrated over the full width of the cavity, this is clearly the second strongest mode. For Fig. 6 we have chosen an axial position for which the variation in spectra with cavity geometry is particularly clear.

It should al:o be noted that, although the overall agreement between simulation and analysis is excellent, numerical deviations may have played some role in choosing between particular resonances. As an example, the fundamental $\mathrm{TM}_{010}$ frequency for a perfectly conducting guide is $w_{010}=0.05236 w_{\mathrm{p}}$ in these units. The numerical. eigenvalue computed on this finite mesh, however, is $w_{010}=0.05201 w_{p}$. Although the discrepancy is well under $1 \%$, such differences make exact spectral distributions suspect where weak resonances are involved. In any case, Fig. 6 clearly illustrates that there is relatively little energy in the nonfundamental modes.

To summarize these results, we find that the frequency of pulse injection strongly determines the dominant cavity mode excited by the beam loading. As predicted by the analytic model, the cavity energy ircreases as the square of the number of pulses, while the fraction of this energy not in the fundamental monotonically decreases. The siug calcuiations, which correspond to $\gamma \rightarrow \infty$, are found to yield the same cavity loading dynamics as fully mobile particle calculations of the accelerator mode. The main features of the radiated spectra are in quantitative agreement with the analysis, but secondary features may be model dependent in the simulations.

Finally, self-excitation of the cavities by intense injected pulses does not appear to have fundamental problens. A practical limitation may prove to be 
viltual cathode formation when too much energy is extracted from the pulse, bit even this condition did not degrade the relative enezgy flow significantly. The efficiency of field accretion was reduced, however. Further work on this concept is apparently needed to assess its ultimate utility.

IV. BEAM BREAKUP INSTABILITY

The beam breakup instability causes the deflection of a charged particle beam in a linear accelerator from its original straight line path by a selfgenerated azimuthal magnetic field $-B_{\theta}$. In a cylindrical $r f$ accelerating cavity the typical beam breakup field is a TM $1 \mathrm{c}$ mode. ${ }^{5}$ This mode is producea by the propagation through the cavity of one or more beam pulses whose centroids are slightly displaced from the cavity axis; its amplitude is proportional to the magnitude of the displacement: Because the field so produced deflects the beam and in turn increases its centroid's displacement from the cavity axis, there is a positive feedback loop which drives the instability.

In this section we calculate the maximum possible radial displacement caused by the bearn breakup instability for a multipulsed relativistic electron beam produced in M-rf-accelerating cavities. We find that a series of 10-kA electron micropulses can be accelerated to $1 \mathrm{GeV}$ electron energies through forty $50-\mathrm{MHz}$ rf cavities each with an accelerating gradient of $14 \mathrm{MV} / \mathrm{m}$ and suffer no more than a 20-fold increase in the magnitude of an initial radial perturbation. Larger accelerating gradients result in even smalier radial displacements.

The amplitude and phase of the ${ }^{T M} 110{ }^{B}{ }_{\theta}$ field produced by the passage of $N$ pulses of duration $\mathrm{T}$, radius $\mathrm{R}_{0}$ and the centroid of each coherently displaced by an amount $\delta$ are straightforwardly calculated from Eq. (15) and given by

$$
B_{\theta 110}(r, \theta, t)=-q_{110}(t) \frac{d}{d r} a_{z 110}(r, \theta) .
$$

Therefore, 


$$
\begin{aligned}
& B_{\theta 110}(r, \theta, t)=\left(\frac{N \rho \delta}{\varepsilon R^{4} w_{110}}\right)\left[\frac{x_{11}^{2} J_{1}^{\prime}\left(x_{11} R_{0} / R\right)}{\pi J_{2}^{2}\left(x_{11}\right)}\right] \cos \theta J_{1}^{\prime}\left(x_{11} r / R\right) \\
& \times\left(\frac{\sin w_{110^{d / 2 c}}}{w_{110^{d / 2 c}}}\right)\left(\begin{array}{c}
\sin w_{110^{T / 2}} \\
-w_{110^{T / 2}}
\end{array}\right)\left(\begin{array}{l}
\sin N \pi w_{110^{/ w_{010}}} \\
N \sin \pi w_{110} / w_{010}
\end{array}\right) \\
& \times \sin w_{110}\left(t-d / 2 c-T / 2-(N-1) \pi / w_{010}\right) .
\end{aligned}
$$

Note that for $\operatorname{small} r$, that is, for $x_{11} r / R \ll 1, J_{2}^{\prime}\left(x_{11} r / R\right) \cong 1 / 2$ and $B_{\theta}$ is independent of $r$.

The radial equation of motion of a nonrotating electron in the middle of the $(\mathrm{N}+1)^{\text {th }}$ pulse is

$$
\ddot{r}=\left(\mathrm{ec} / \mathrm{m}_{\gamma}\right) \mathrm{B}_{\theta}(\mathrm{t}) \text {, }
$$

where $\gamma$ is assumed constant throughout a single cavity. Integrating this equation over the time spent in one cavity, the total radial displacement and velocity per cavity $r_{\dot{f}}$ and $\dot{r}_{f}$ is found

$$
\begin{aligned}
\dot{r}_{f} & =\dot{r}_{i}+\left(A^{-} \xi w_{110} / \gamma\right) \sin w_{110} d / 2 c\left\{\sin \left[(N+1) \pi w_{110} / w_{010}\right]\right\} \\
r_{f} & =r_{i}+\dot{r}_{i} d / c+\left(A^{\prime} \xi w_{110} d / 2 c \gamma\right)\left\{\cos \left[w_{110}\left(\pi(N+1) / w_{010}-d / 2 c\right)\right]\right. \\
& \left.-\left(2 c / w_{110} d\right) \sin w_{110} d / 2 c \cos \left[(N+1) \pi w_{110} / w_{010}\right]\right\}
\end{aligned}
$$

where $A^{\prime}=\left(\frac{N \rho e}{\varepsilon R m c^{2}}\right)\left(\frac{2 J_{1}^{\prime}\left(x_{11^{2}} / R\right)}{\pi x_{11} J_{2}^{2}\left(x_{11}\right)}\right) \cos \theta J^{\prime}\left(x_{11} r / R\right)$

$$
\times\left(\frac{\sin w_{110^{d / c 2}}}{w_{110^{d / 2 c}}}\right)\left(\frac{\sin w_{110^{T / 2}}}{w_{110^{T / 2}}}\right)\left(\frac{\sin N \pi w_{110^{/}} / w_{010}}{N \sin \pi w_{110^{/}} w_{010}}\right)
$$


and $r_{i}$ and $r_{i}$ are initial values upon entering the cavity.

A set of simple recursion relations that determine the maximum possible radial displacement and velocity of an electron in the $(N+1)^{\text {th }}$ pulse as it leaves the $M^{\text {th }}$ cavity $r_{M}$ and $\dot{r}_{M}$ can be derived from Eqs. (33)-(34) by using the following assumptions.

1) The radial displacement of the previous $N$ pulses in the $M^{\text {th }}$ cavity is $\mathbf{r}_{M-1}$.

2) The factors within the curly brackets are set equal to one.

3) $\operatorname{Sin} N \pi w_{110} / w_{010}=1$.

4) $\cos \theta=i$.

5) The $\gamma$ in the $M^{\text {th }}$ cavity, $\gamma_{M}$, is given by $\gamma_{M}=\gamma_{i}+(M-1) \gamma_{0}$, where $\gamma_{i}$ is the injection energy and $\gamma_{0}$ is the energy increment per cavity.

6) $x_{11} R_{0}$ and $x_{11} r \ll R$.

Assumptions $1,3,4,5$, and 6 always overestimate the instability. Assumption 2 also overestimates the instability whenever $2 c / w_{110} d \leqq 1$, which is the case in the following numerical examples. Note that assumptions 2 and 3 remove the dependence on $N$, the number of pulses. The recursion relations are therefore

$$
\dot{r}_{M}=\dot{r}_{M-1}+A w_{110} \sin \left(w_{110} d / 2 c\right) r_{M-1} /\left[\left(\gamma_{i} / \gamma_{0}\right)+M-1\right]
$$

and

$$
\left.r_{M}=r_{M-1}+\dot{r}_{M-1} d / c+\left(A w_{110} d / 2 c\right) r_{M-1} /\left[\gamma_{i} / \gamma_{0}\right)+M-1\right],
$$

where

$$
\begin{aligned}
A= & \left(\frac{\rho e}{\varepsilon R m \gamma_{0} c^{2}}\right)\left[\frac{2 J_{1}^{\prime}\left(x_{11} R_{0} / R\right)}{\pi \chi_{11} J_{2}^{2}\left(x_{11}\right)}\right]\left|\frac{\sin w_{110^{d / 2 c}}}{w_{110} d / 2 c}\right| \\
& \left|\frac{\sin w_{110^{T}} T / 2}{w_{110^{T / 2}}}\right|\left|\frac{1}{\sin \pi w_{110} / w_{010}}\right|
\end{aligned}
$$


Solutions to Eqs. (35)-(36) in terms of the radial deflection after the $M^{\text {th }}$ cavity $r_{M}$ normalized to the size of an initial displacement $r_{0}$ are shown in Fig. 7 for two cases of interest. For these cases we have used parameters for a $50-\mathrm{MHz}$ rf cavity such as those used in PHERMEX $(\mathrm{d}=2.6 \mathrm{~m}, \mathrm{R}=2.3 \mathrm{~m})$, a 10-kA micropulse current $\left(T=2.5 \mathrm{~ns}, \rho=2.510^{-5}\right.$ coulombs/micropulse), accelerating gradients 14 and $34 \mathrm{MV} / \mathrm{m}$, and an injection kinetic erergy of $3.07 \mathrm{MV}$. A 40 cavity accelerator of this type would correspondingly accelerate electrons to $1.0 \mathrm{GeV}$ or $2.5 \mathrm{GeV}$. Note that the worst case, $14 \mathrm{MV} / \mathrm{m}$ and $M=40$, gives only a 20-fold increase in the magnitude of a presumably small initial radial displacement.

\section{ACKNOWLEDUMENT}

The authors gratefully acknowledge many helpful discussions with Richard Cooper. This work was supported by the Air Force Office of Scientific Research.

\section{REFERENCES}

1. D. C. Moir, R. J. Faehl, B. S. Newberger, and L. E. Thode, "Suitability of High-Current Standing-Wave Linac Technology for Ultra-Relativistic Electron Beam Propagation Experiments," Los Alamos National Laboratory report LA-8645-MS (1981).

2. D. Venable, Ed., "PHEPMEX: A Pulsed High-energy Radiographic Machine Emitting X-Rays," Los Alamos Scientific Laboratory report LA-3241 (May $1967)$.

3. Memorandum from P. J. Tallexico to T. J. Boyd, subject: Kilpatrick's Criterion, July $10,1974$.

4. W. D. Kilpatrick, "Criterion for Vacuum Sparking Designed to Include Both rf and dc," Rev. Sci. Instrum. 28, 824 (1957).

5. R. Helm and G. Loew, "Beam Breakup," Chap. B.1.4 in Linear Accelerators, P. M. Lapostolle and A. L. Septier, Eds. (North Holland Publishing Company, Amsterdam, 1970).

6. E. U. Condon, "Forced Oscillations in Cavity Resonators," J. Appl. Phys. $12,129(1941)$.

7. M. Friedman, "Autoacceleration of an Intense Relativistic Electron Beam," Phys. Rev. Letters 31, 1107 (1973). 


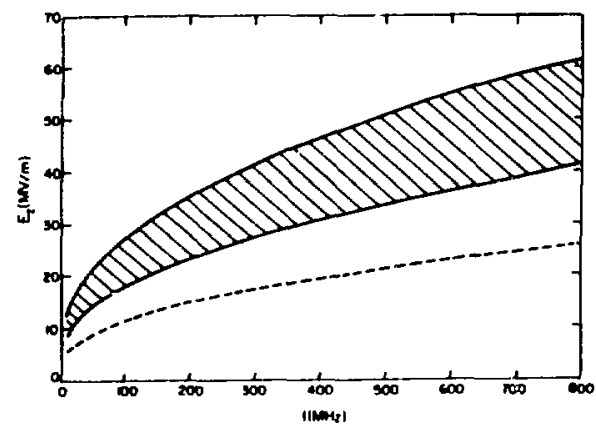

Fig. 2.

Energy radiated into cavity modes $\Sigma_{2} \mathrm{U}_{\lambda}$ as a function of pulse number $\mathrm{N}$. The open boxes represent the selfconsistent simulation results, the closed circles the "slug" simulation results, and the $x^{\prime} s$ the analytic theory.

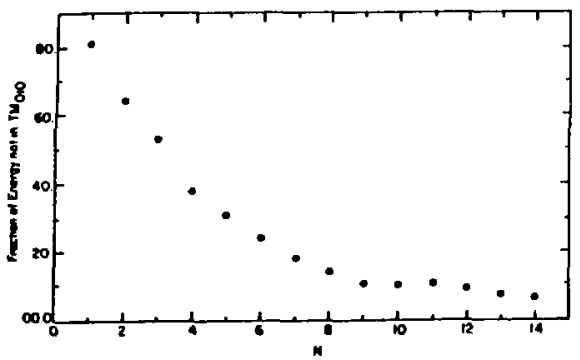

Fig. 1 .

Sparking limits as a function of frequency. The two solid lines are $E_{Z}(M V / m)$ for $K=3$ (upper line) and $K=2$ (lower line). The dashed line is $E_{K}$.

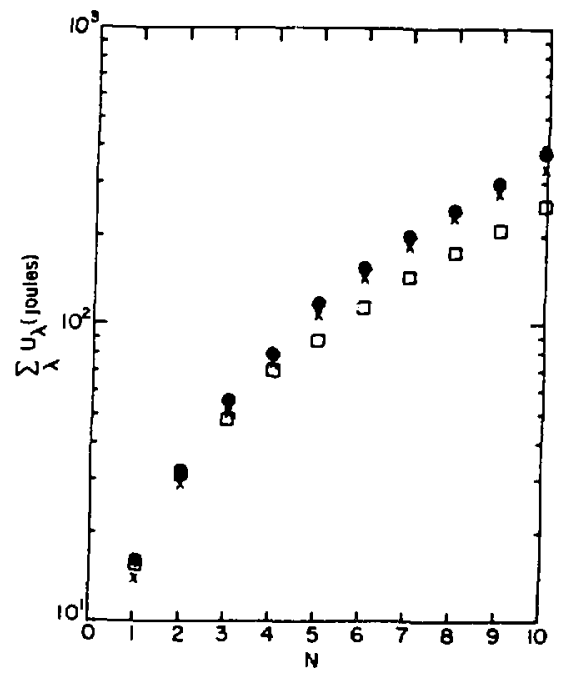

Fig. 3.

Relative fraction of field energy in cavity fundamental $\omega_{010}$ as a function of pulse number $\mathbf{N}$ for slug simulation $(\gamma=\infty) ; I_{\max }=9.2 \mathrm{kA}, I_{\text {ave }}=0.6 \mathrm{kA}$, $T=14.1 \omega_{\mathrm{p}}$. 


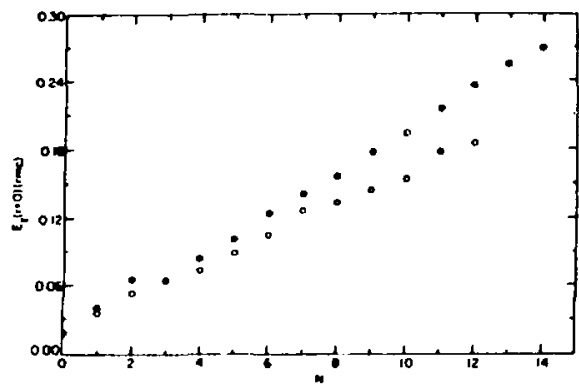

Fig. 4.

Comparison of rms magnitude of $E_{z}$ in slug $\left(\gamma_{0}=\infty\right.$, closed circles) and particle $\left(Y_{0}=10.0\right.$, open circles) simulations as a function of pulse number $\mathrm{N}$; $\mathrm{I}_{\max }=9.2 \mathrm{kA}, \mathrm{I}_{\text {ave }}=0.6 \mathrm{kA}$, $\therefore=1.0 \mathrm{\omega}_{\mathrm{p}}$. Because of partial pulse injection initially on particle calculations, pulse number is shifted by one relative to actual time for plotting.

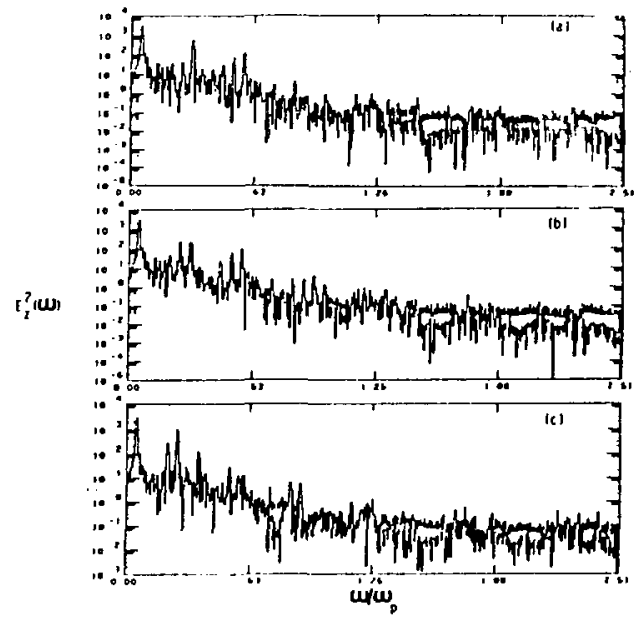

Fig. 6 .

$E_{z}$ spectrum on axis at $z=0.8 \mathrm{~d}$ after 10 pulses; (a) $d / R=1.105$, (b) $d / R=$ 1.114 , (c) $\mathrm{d} / \mathrm{R}=1.159$.

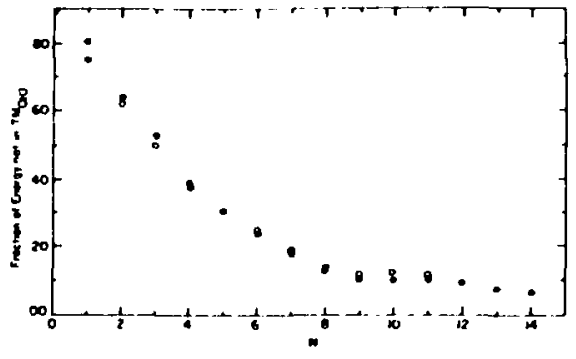

Fig. 5.

Comparison of relative fraction of field energy not in $\mathrm{TM}_{010}$ for same calculations as Fig. 4.

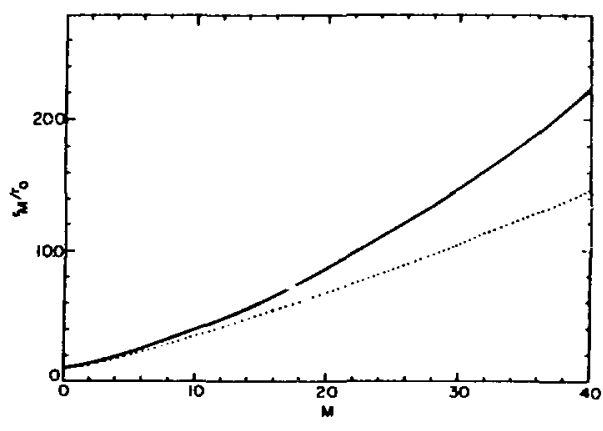

Fig. 7.

Maximum radial displacement of the beam centroid after leaving the $\mathrm{M}^{\text {th }}$ cavity $r_{M}$ normalized to the initial perturbation $r_{0}$ versus $M$ as determined from Eqs. (35)-(36). Peak accelerating gradients per cavity are $14 \mathrm{MV} / \mathrm{m}$ (solid) and $34 \mathrm{MV} / \mathrm{m}$ (dotted). other parameters are $\mathrm{R}=2.3 \mathrm{~m}, \mathrm{~d}=2.6 \mathrm{~m}$, $\mathrm{T}=2.5 \mathrm{~ns}, \rho=2.510^{-5}$ coulombs $/$ micropulse and $\gamma_{i}=7.0$. 